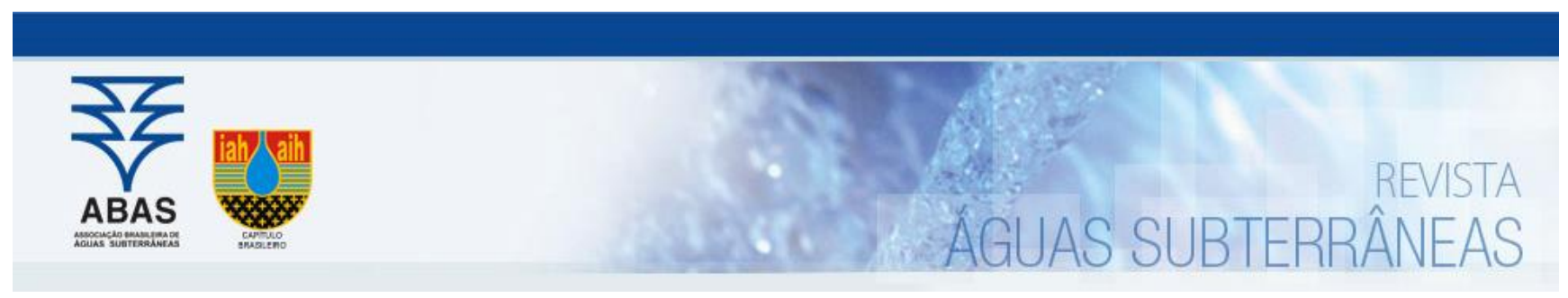

Artigos

\title{
Utilização de métodos de campo e laboratoriais para estimação de propriedades hidrodinâmicas do solo
}

\author{
Use of field and laboratory methods to estimate soil hydrodynamic \\ properties
}

\author{
Gercica Macêdo1; Willames Soares ${ }^{1}$ \\ 1 Universidade de Pernambuco (UPE), Recife, PE \\ $\bowtie$ gercica.macedo@gmail.com,was@poli.br
}

Palavras-chave:

Drenagem urbana.

Curva de retenção.

Curva de condutividade hidráulica.
Método Beerkan.

\begin{abstract}
Resumo
O solo é de suma importância na drenagem urbana, pois auxilia na retenção da água proveniente das chuvas, através da infiltração, e reduz o escoamento superficial que tem como consequência as inundações urbanas. Determinar as propriedades hidrodinâmicas do solo é essencial para compreender esses processos. As propriedades hidrodinâmicas do solo são determinadas pela curva de retenção da água no solo e a curva de condutividade hidráulica. A determinação das propriedades hidrodinâmicas do solo requer ensaios de campo e de laboratório bastante dispendiosos, oneram e demandam um longo tempo de execução. Desse modo, o objetivo desse trabalho é determinar as propriedades hidrodinâmicas do solo pelos métodos Beerkan e Função de Pedotransferência. $O$ estudo foi realizado no Instituto Federal de Pernambuco, Campus Recife, constituindo-se das etapas de coleta de amostras, ensaios de campo e ensaios de laboratório. 0 perfil do solo dos pontos amostrais do local de estudo foi classificado como franco arenoso. As propriedades hidrodinâmicas do solo obtidos pelo método da Função de Pedotransferência estão coerentes com os observados na literatura e as curvas de retenção do solo e de condutividade hidráulica, apresentaram comportamento semelhante em todos os pontos analisados. Para o método Beerkan, foram determinados os parâmetros de forma e de normalização, apresentando valores aceitáveis comparados aos da literatura e além disso, as curvas de infiltração ajustadas, as curvas de retenção e condutividade hidráulica nos pontos analisados tiveram comportamentos semeIhantes entre elas.
\end{abstract}

Abstract

Keywords:

Urban drainage.

Retention curve.

Curve of hydraulic conductivity.

Beerkan Method.

Revisado por pares.

Recebido em: 07/03/2020.

Aprovado em: 19/05/2020.
Soil is of great importance in urban drainage, as it assists in the retention of water from the rains through infiltration and reduces the surface runoff that has as a consequence urban floods. Determining the hydrodynamic properties of the soil is essential to understanding these processes. The hydrodynamic properties of the soil are determined by the water retention curve in the soil and the hydraulic conductivity curve. The determination of the hydrodynamic properties of the soil requires field and laboratory tests that are expensive, costly and require a long execution time. Thus, the objective of this work is to determine the hydrodynamic properties of the soil by the Beerkan and Pedotransfer Function methods. The study will be carried out at the Federal Institute of Pernambuco, Recife Campus, constituting the stages of sample collection, field trials and laboratory tests. The soil profile of the sampling points at the study site was classified as sandy loam. The hydrodynamic properties of the soil obtained by the Pedotransfer Function method are consistent with those observed in the literature and the soil retention and hydraulic conductivity curves, showed similar behavior in all analyzed points. For the Beerkan method, shape and normalization parameters were determined, presenting acceptable values compared to those in the literature and, in addition, the adjusted infiltration curves, retention curves and hydraulic conductivity at the analyzed points had similar behaviors between them.

\section{INTRODUÇÃO}

Os alagamentos são frequentes nas cidades mal planejadas ou que crescem desordenadamente, já que a realização de obras de drenagem e de esgotamento de águas pluviais é deixada em segundo plano (FUNASA, 2006). Problemas de ordem ambiental e social são consequências da urbanização acelerada sem planejamento. Dentre os problemas ambientais está a impermeabilização do solo, reduzindo a infiltração da água pluvial e aumentando o escoamento superficial, o que tem como consequência direta a ocorrência de alagamentos urbanos.
Segundo Holanda e Soares (2019) o processo de urbanização interfere nos elementos do ciclo hidrológico, alterando a infiltração, vazão e evaporação da água da chuva. Com a existência desse tipo de solo, surgem problemas para aquíferos e fontes de água subterrânea, especialmente em grandes centros urbanos, pois o suprimento de água provém da água que se infiltra da superfície para as camadas inferiores do solo. Além disso, uma grande concentração urbana é considerada um problema que favorece as inundações devido ao uso inadequado da terra e à ocupação do espaço, bem como pelo manejo insuficiente da drenagem 
urbana.

Sendo assim, é necessário que áreas livres de construção existam para amenizar os efeitos causados pela urbanização acelerada, como reduzir ou evitar alagamentos urbanos. 0 solo natural apresenta características que atenuam a infiltração e condução da água em seu interior, causando um maior escoamento superficial, característico da região.

Com a existência de áreas livres de construção, solo natural, é necessário identificar a capacidade de armazenamento e a facilidade com que a água é conduzida através do solo, sendo determinado através das propriedades hidrodinâmicas do solo. Segundo Grigolon (2013), as propriedades hidrodinâmicas do solo são determinadas pela curva de retenção da água no solo e a curva de condutividade hidráulica. As características de retenção da água e da condutividade hidráulica do solo avaliam, respectivamente, sua capacidade de armazenamento e a facilidade com que a água é conduzida através dele. A determinação das propriedades hidrodinâmicas do solo requer ensaios de campo e de laboratório bastante dispendiosos, onerando e demandando um longo tempo de execução. Por esses motivos, levou alguns pesquisadores a desenvolverem métodos mais simples que requerem dados do solo prontamente disponiveis, usuais e de baixo custo (WAGNER et al., 1998; MINASNY et al. 1999; MINASNY; MCBRATNEY, 2002).

Vários modelos foram desenvolvidos na literatura para descrever o comportamento da curva de retenção da água no solo (GARDNER, 1958; BROOKS; COREY, 1964; VAN GENUCHTEN, 1980; FREDLUND; XING, 1994). Os modelos mais abordados na literatura são:

- Brooks e Corey (1964):

$\theta(h)=\frac{\left(\theta-\theta_{r}\right)}{\left(\theta_{s}-\theta_{r}\right)}=\left[\frac{h}{h_{e a}}\right]^{-2 \lambda_{t p}}$

para $h \leq h_{e a}$

$\operatorname{com} \theta=\theta_{s}$ para $h_{e a} \leq h \leq 0$

- Van Genuchten (1980):

$\theta(h)=\frac{\left(\theta-\theta_{r}\right)}{\left(\theta_{s}-\theta_{r}\right)}=\left[1+\left(\frac{h}{h_{g}}\right)^{n}\right]^{-m}$

Com $m=1-\frac{1}{n}, \mathrm{n}>1$, Mualem (1976) e

$$
\begin{aligned}
& m=1-\frac{2}{n}, \mathrm{n}>2, \text { Burdine (1953) } \\
& h_{g}=\frac{1}{\alpha}
\end{aligned}
$$

Sendo: $\theta(\mathrm{h})$ a umidade efetiva; $\theta_{s}$ a umidade saturada $\left[\mathrm{cm}^{3} . \mathrm{cm}^{-3}\right] ; \theta_{r}$ a umidade residual $\left[\mathrm{cm}^{3} . \mathrm{cm} 3\right] ; h$ o potencial matricial [cm]; $h_{e a}$ e $h_{g}$ parâmetros de ajuste que dependem da estrutura do solo [cm] e $\lambda_{t p}, \alpha$, m e n parâmetros de forma que dependem principalmente da textura.

Segundo Reichardt e Timm (2004), a condutividade hidráulica é, obviamente, afetada pela estrutura e textura do solo, sendo maior em solo altamente poroso, fraturado ou agregado e menor em solos densos e compactados. A condutividade hidráulica tem como propriedades mais importantes do solo a forma de suas partículas, a superfície específica, a porosidade, a estrutura, a compactação, ou seja, todas as propriedades que refletem a geometria porosa dos solos (ANGELOTTI NETTO; FERNANDES, 2005).

Vários modelos foram desenvolvidos na literatura para descrever o comportamento da condutividade hidráulica:

- Brooks e Corey (1964):

$K(\theta)=K_{s}\left(\frac{\theta-\theta_{r}}{\theta_{s}-\theta_{r}}\right)^{\eta}$

Sendo: $\left(\frac{\theta-\theta_{r}}{\theta_{s}-\theta_{r}}\right)$ considerada a saturação efetiva; $\eta$ é um parâmetro de forma, definido pela equação 4 abaixo.

$\eta=\frac{2}{m n}+2+p$

Sendo: $\mathrm{m}$ e $\mathrm{n}$ são parâmetros de forma, $\mathrm{p}$ é o fator de tortuosidade, definido por $p=1$ para o modelo de Burdine (1953), $p=0,5$ para o modelo de Mualem (1976) ou $p=0$ para o modelo de Chids e Collis-George (1950).

- Van Genuchten (1980):

$K(\theta)=K_{S}(\Theta)^{2}\left[1-\left(1-(\Theta)^{1 / m}\right)^{m}\right]$

Com $m=1-\frac{K}{n}, \mathrm{k}=2$, Burdine (1953)

$K(\theta)=K_{S}(\Theta)^{1 / 2}\left[1-\left(1-(\Theta)^{1 / m}\right)^{m}\right]^{2}$

Com $m=1-\frac{K}{n}, \mathrm{k}=1$, Mualem (1976).

Sendo: $K_{S}$ é a condutividade hidráulica saturada do solo [cm.s 1].

Segundo Lassabatere et al. (2006), vários métodos têm sido desenvolvidos para determinar as propriedades hidrodinâmicas do solo. Os mais simples requerem informação só prontamente disponíveis, tais como a distribuição de tamanho de partículas e características físicoquímicas ou medições de campo simples (JARVIS et al., 2002). Os mais sofisticados exigem o experimental completo na determinação da curva de retenção de água $\theta(h)$ e a condutividade hidráulica $\mathrm{K}(\theta)$ utilizando técnicas de laboratório (RAIMBAULT, 1986; MALLANTS et al., 1997).

De acordo com Bruning et al. (2019) é crescente a utilização de Funções de Pedotransferência (FPT) que utilizam a análise textural para estimativa dos parâmetros empíricos dos modelos. As FPT permitem a previsão dos parâmetros hidrodinâmicos de Van Genuchten e da condutividade hidráulica saturada através da utilização de dados texturais dos solos como entrada, tornando assim, uma ferramenta útil para os locais com limitações de dados.

Segundo Tomasella e Hodnett (2004), os fatores importantes para o desempenho de uma FPT são as características pedológicas dos solos que foram utilizadas para o desenvolvimento das suas equações, pois as diferenças entre esses e os solos que se queiram estimar as propriedades podem ser a causa do baixo desempenho de um determinado modelo. Os autores relatam que essa pode ser a causa da tendência de Funções de Pedotransferência modeladas sobre solos de clima temperado serem mal sucedidas quando aplicadas para solos de clima tropical. Nesse mesmo sentido, de acordo com Costa e Soares (2019), verificou-se que a 
origem dos solos utilizados para o desenvolvimento das equações é fator influenciador na eficiência, ou seja, modelos aplicados a solos com características pedológicas semelhantes aos utilizados na sua criação tendem a obter melhores resultados.

A metodologia Beerkan foi idealizada por Haverkamp et al. (1994). o Beerkan é um método que propõe a estimativa dos parâmetros das curvas de retenção $\theta(\mathrm{h})$ e de condutividade hidráulica do solo $K(\theta)$, descritas respectivamente pelas equações de Van Genuchten (1980) e Brooks e Corey (1964) (HAVERKAMP et al., 1998; BRAUD et al., 2005; LASSABATÈRE et al., 2006). Neste método, $\theta(\mathrm{h})$ e $\mathrm{K}(\theta)$ podem ser descritas, analiticamente, por cinco parâmetros: dois de forma ( $\mathrm{m}$ ou $\mathrm{n}$ e $\eta$ ), relacionando principalmente com a textura, e três de normalização $\left(\theta_{\mathrm{s}}, \mathrm{K}_{\mathrm{s}}\right.$ e $\left.\mathrm{h}_{\mathrm{g}}\right)$, dependentes da estrutura do solo (SOUZA et al., 2008).

O método Beerkan é um método de medição de campo e laboratorial. Os parâmetros de forma são obtidos a partir da curva de distribuição dos tamanhos das partículas $F(D)$ e da porosidade, enquanto os parâmetros de normalização são determinados a partir de experimentos de infiltração. Esse método propõe a obtenção da curva de infiltração acumulada em função do tempo, com um infiltrômetro a disco ou com um infiltrômetro de anel simples (CAVALCANTI, 2012).

Diante do exposto, este trabalho propõe estimar as propriedades hidrodinâmicas do solo do Instituto Federal de Pernambuco, Campus Recife, por meio da aplicação dos métodos de medição Função de Pedotransferência e Beerkan.

\section{MATERIAIS E MÉTODOS}

O local de estudo para a coleta das amostras e ensaios de campo foi o Instituto Federal de Educação Ciência e Tecnologia de Pernambuco (IFPE), Campus Recife, localizado no bairro da Cidade Universitária na região metropolitana, situado no litoral do estado de Pernambuco (Figura 1). O IFPE foi escolhido pelo solo do local se assemelhar às características do solo da região próxima e por ser representativo dos outros solos. Além disso, o solo do IFPE tem características urbanas, pois está localizado em uma área urbanizada com edificações próximas.

Figura 1- Localização do IFPE

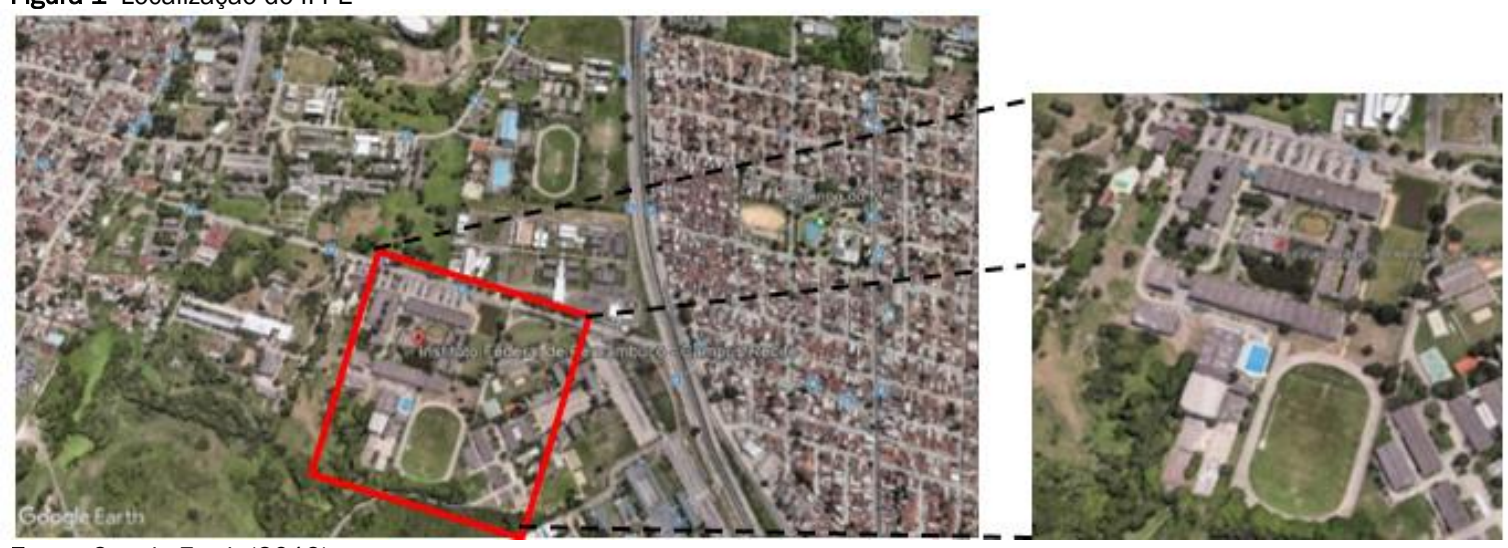

Fonte: Google Earth (2019).

A etapa de coleta de amostras foi realizada em 9 pontos distintos do IFPE (em vermelho), Campus Recife, e ensaio de infiltração em 3 pontos distintos (P1, P2, P3). A distância entre os pontos foi de $3 \mathrm{~m}$ e os pontos do ensaio de infiltração foram localizados no centro em relação aos pontos de coleta das amostras para o ensaio de granulometria (em amarelo), conforme Figura 2.

Figura 2- Visão aérea dos pontos de coleta e do ensaio de infiltração no IFPE

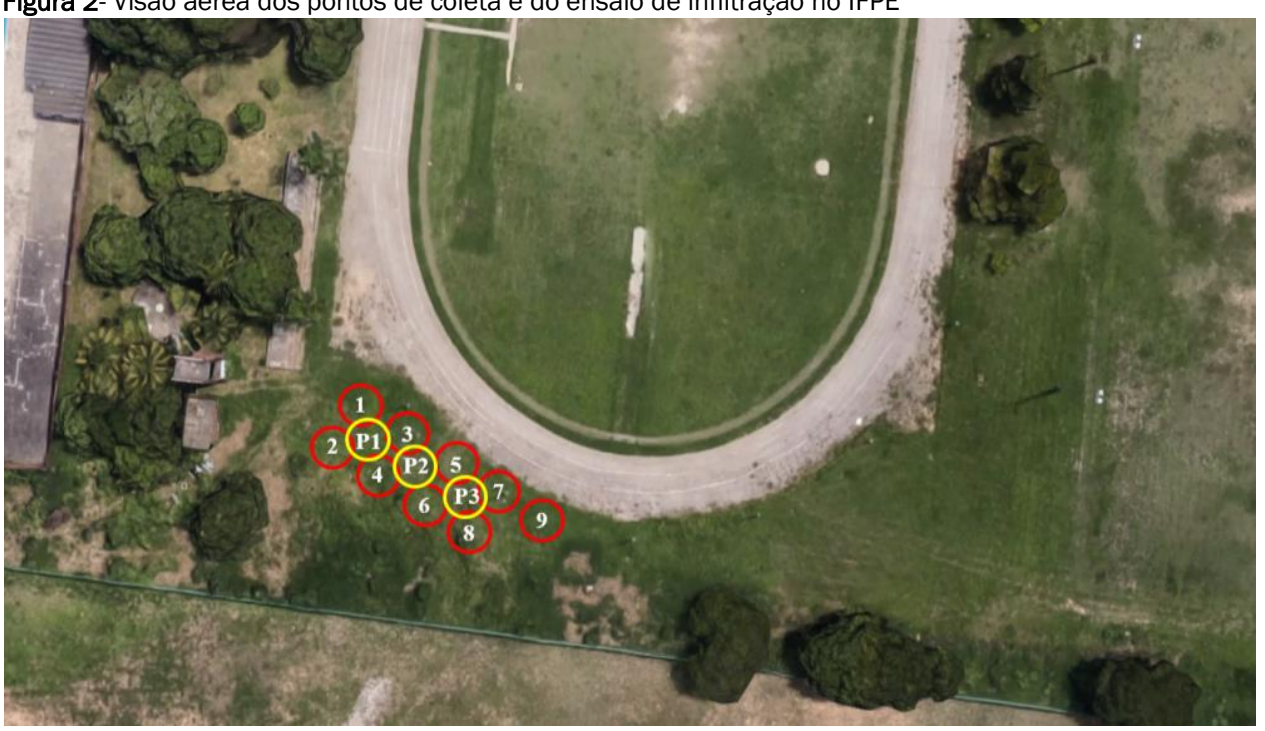

Fonte: Autores 
O ensaio de granulometria foi realizado por uma combinação de sedimentação e peneiramento, de acordo com as instruções da NBR 7181 (ABNT, 2016b).

\subsection{Aplicação do Método Função de Pedotransferência}

Para a Determinação das propriedades hidrodinâmicas do solo pelo Método Função de Pedotransferência foi utilizado o modelo de Van Genuchten (1980) para o cálculo da curva de retenção da água no solo e a condutividade hidráulica, com a condição k=1 (Mualem, 1976).

A Função de Pedotransferência utilizada para calcular os parâmetros do modelo de Van Genuchten (1980) é a do modelo de Barros et al. (2013), por se tratar de um estudo significativo, que utilizou solos do Nordeste brasileiro.

No estudo de Barros et al. (2013) dois tipos de Funções de Pedotransferência (FPT) foram ajustadas e avaliadas. A equação escolhida utiliza apenas duas variáveis do solo: teores de areia e argila, definida pela equação 7 :

PTF-2v $\quad y_{i}=\beta_{i, 0}+\beta_{i, 1} A+\beta_{i, 2} G+\varepsilon_{i}$

Sendo: A é o teor de areia [kg.kg-1], G é o teor de argila [kg.kg1], O é o teor de matéria orgânica [kg.kg-1], $\rho_{s}$ é a densidade aparente [g.cm-3], $y_{i}$ corresponde aos parâmetros do modelo de Van Genuchten e $\beta$ representa o parâmetro referente ao teor de cada variável do solo, especificados na metodologia de Barros et al. (2013). o $\varepsilon$ representa o erro aleatório associado a cada observação.

Para a condutividade hidráulica saturada (Ks), será estimada através das porcentagens de areia e argila provenientes das amostras do solo analisado de acordo com a equação proposta por Cosby et al. (1984), conforme equação 8:

$K_{S}=7,05556 \cdot 10^{-6} \cdot 10^{(-0,6+0,0126 A-0,0064 G)}$

Os parâmetros obtidos seguem a hipótese de Mualem, e os parâmetros obtidos da literatura seguem a hipótese de Burdine. Logo, foi necessário ajustar os parâmetros obtidos nesse estudo para a mesma hipótese dos demais estudos para realizar uma comparação coerente. A conversão da condição de Mualem (1976) para a condição de Burdine (1953) foi realizada através do software RETC (RETention Curve).

\subsection{Aplicação do Método Beerkan}

Para a determinação das propriedades hidrodinâmicas do solo a partir do método Beerkan, a curva de retenção da água $\theta(h)$ e a condutividade hidráulica $K(\theta)$ são descritas, respectivamente, pelos modelos de Van Genuchten (1980), que trata da curva de retenção da água, e de Brooks e Corey (1964) que trata da Curva de Condutividade Hidráulica, usando o modelo de Mualem.
Nessas equações, os parâmetros de forma ( $m$ ou $n$ e $\eta$ ) são obtidos através da curva de distribuição dos tamanhos das partículas $F(D)$ e da porosidade, e os parâmetros de normalização $\left(\theta_{\mathrm{s}}, \mathrm{K}_{\mathrm{s}}\right.$ e $\left.\mathrm{h}_{\mathrm{g}}\right)$ foram determinados por intermédio de ensaios de infiltração.

Para a determinação da curva de distribuição do tamanho das partículas $F(D)$ foram realizados ensaios de granulometria de acordo com a NBR 7181 (ABNT, 2016b). Sendo assim, com a determinação da curva granulométrica e dos valores $\theta_{0}, \theta_{\mathrm{s}} \mathrm{e}$ $\rho_{s}$, e a execução do ensaio de infiltração, foram identificados os parâmetros de forma e normalização.

A etapa de coleta de amostras e ensaios de campo foi constituído pelo ensaio de infiltração de anel simples, remoção de amostras para identificar a umidade inicial e final do solo, amostras para a determinação da granulometria do solo e amostra para a determinação das densidades. Os ensaios de campo foram realizados no mesmo local onde foi coletado as amostras de solo para a realização da granulometria.

O ensaio de campo de infiltração de anel simples foi realizado utilizando um cilindro metálico de $15,47 \mathrm{~cm}$ de diâmetro cravado na superfície do solo à uma profundidade de aproximadamente $1 \mathrm{~cm}$ para evitar perdas de água laterais durante o ensaio de infiltração, de acordo com a metodologia desenvolvida por Braud et al. (2005) e Lassabatere et al. (2006).

Em seguida, foi vertido no anel um volume de água conhecido continuamente até que fosse completamente infiltrado no solo. O segundo volume conhecido de água foi adicionado ao cilindro novamente. Foram cronometrados os tempos que os volumes conhecidos de água levam para ser infiltrados no solo. As leituras pararam quando as velocidades de infiltração tenderam a ser constante.

A umidade inicial e saturada do solo e densidade do solo foram determinadas através da coleta de amostras no local do estudo. Para determinar a umidade saturada, foram seguidas as orientações do Manual de Métodos de Análise de Solo da Empresa Brasileira de Pesquisa Agropecuária (EMBRAPA, 1997). Para a determinação da densidade do solo, foi utilizada as mesmas amostras indeformadas coletadas para o ensaio da umidade saturada e para a determinação da densidade das partículas, foram utilizadas as amostras coletadas para a granulometria.

Para determinar os parâmetros de forma e de normalização, seguiu-se a metodologia de Lassabatere et al. (2006).

\section{Resultados e discussão}

\subsection{Granulometria}

A partir das curvas granulométricas obtidas para cada ponto, foi possivel determinar as frações de areia, silte e argila. Dentre as representações das curvas na Figura 3, é possível perceber comportamento semelhante em todos os pontos. 
Figura 3- Curvas granulométricas dos pontos analisados

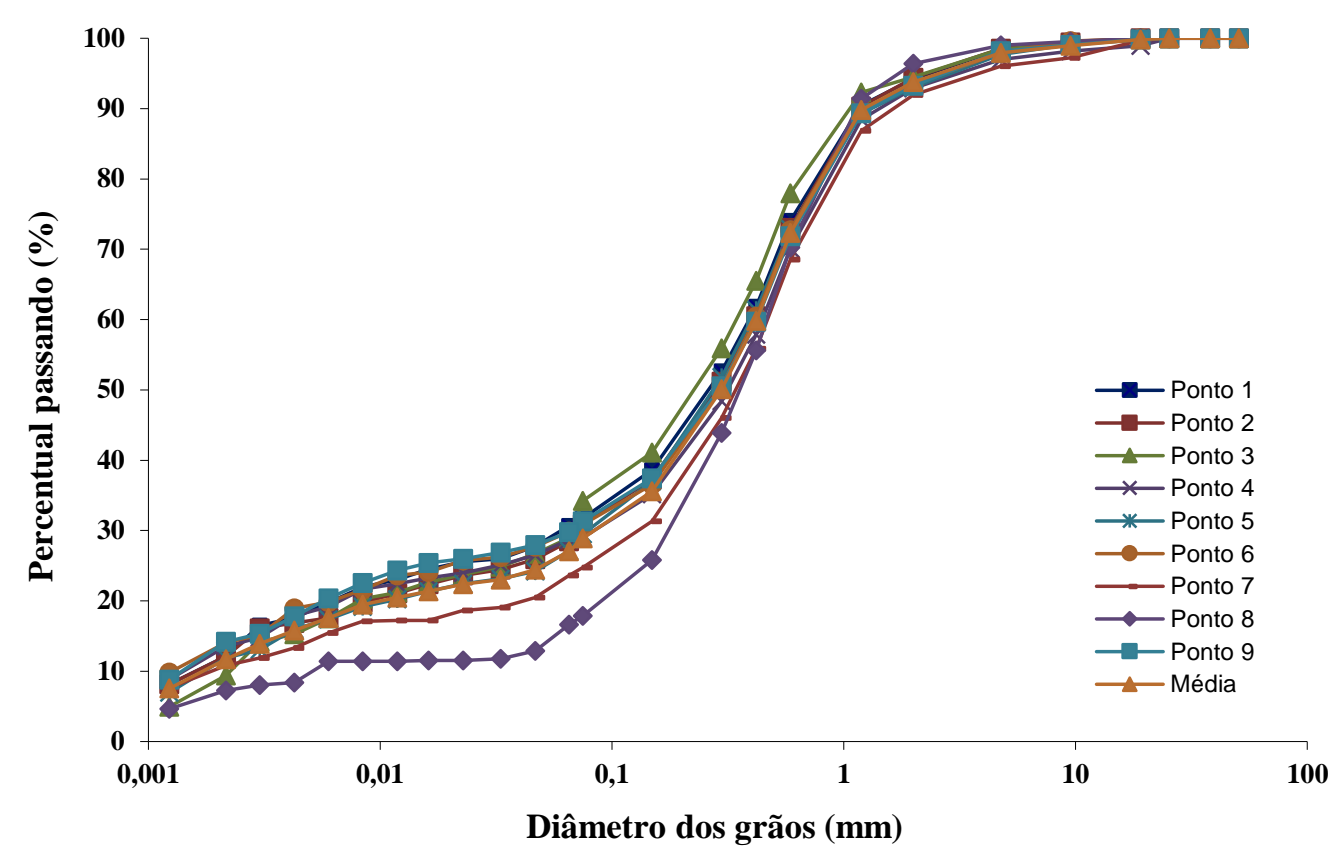

Fonte: Autores

0 ponto 8 foi um dos que representou maior distinção em comparação aos demais. A partir dos percentuais de argila, silte e areia determinados nos ensaios de granulometria foi possível obter as curvas granulométricas para cada ponto.

É possível identificar que a textura do solo se apresentou como de maioria franco arenosa. Apenas o ponto 8 demonstrou uma maior variação dos percentuais de argila, silte e areia, com classe textural areia franca, devido ao menor percentual de silte e argila, comparando-se aos demais. 0 ponto 8 está localizado próximo a um muro no IFPE e um dos motivos para essa diferença pode ser a intervenção de mate- rial de construção no solo, modificando a classe textural.

\subsection{Determinação das Curvas de Retenção e Condutividade do Solo pelo método da FPT}

A partir dos teores de argila, silte e areia, foi possível determinar os parâmetros de Van Genuchten (1980) com a hipótese de Mualem, através da Função de Pedotransferência, de Barros et al. (2013), e a condutividade hidráulica saturada estimada através da equação de Cosby et al. (1984). Os valores obtidos são apresentados na Tabela 1.

Tabela 1 - Análise estatística dos parâmetros de Van Genuchten

\begin{tabular}{ccccc}
\hline Análise Estatística & $\alpha(1 / \mathrm{m})$ & $\mathrm{n}$ & $\theta_{\mathrm{r}}\left(\mathrm{cm}^{3} / \mathrm{cm}^{3}\right)$ & $\theta_{\mathrm{s}}\left(\mathrm{cm}^{3} / \mathrm{cm}^{3}\right)$ \\
\hline Mínimo & 4,82 & 1,69 & 0,03 & 0,34 \\
Máximo & 6,40 & 1,78 & 0,08 & 0,38 \\
Média & 5,36 & 1,72 & 0,07 & 0,37 \\
Desvio padrão & 0,52 & 0,03 & 0,02 & 0,01 \\
\hline
\end{tabular}

\section{Fonte: Autores}

O parâmetro $\alpha$ variou de 4,82 a 6,40, tendo pequena variação, confirmado no desvio padrão de 0,52 , com média de 5,36 , sendo mais próximo do valor mínimo. 0 parâmetro $n$ variou de 1,69 a 1,78, com pouca variação, confirmado no desvio padrão de 0,03 , com média de 1,72 , sendo mais próximo do

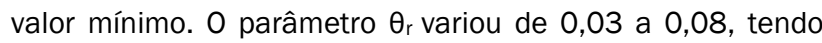
pequena variação, confirmado no desvio padrão de 0,02, com média de 0,07 , sendo mais próximo do valor máximo. 0 parâmetro $\theta_{\mathrm{s}}$ variou de 0,34 a 0,38 , tendo pouca variação, confirmado no desvio padrão de 0,01, com média de 0,37, sendo mais próximo do valor máximo.

Para os parâmetros $\alpha$ e $n$, as médias foram mais próximas dos valores mínimos, pois a maioria dos valores se aproximam do valor mínimo, diferente do ponto 8 que tem valores maiores, destoando um pouco do comportamento dos demais pontos. Para os parâmetros $\theta_{\mathrm{r}}$ e $\theta_{\mathrm{s}}$, as médias foram mais próximas dos valores máximos, pois a maioria dos valores se aproximam do valor máximo, diferente do ponto 8 que tem valores menores. Esses valores são confirmados na literatura através de Reichardt e Timm (2004) que menciona que a umidade saturada e residual para solos arenosos são menores que para solos argilosos.

As curvas de retenção do solo mostram o comportamento da umidade subordinado à pressão, sendo descritas pela equação 2, proposta por Van Genuchten. Para cada curva de retenção do solo, representa a média das 3 amostras de cada 
ponto e a média de todos os pontos (Figura 4), onde as abscissas estão representadas pelas umidades do solo e nas ordenadas a variação da pressão.

É possível perceber que apesar dos diferentes valores de umidade das amostras, os comportamentos referentes à Figura 4 são semelhantes. Todas as amostras coletadas são compostas em maior percentual por areia, sendo assim é possível inferir que o solo estudado possui rápida infiltração, sendo saturado rapidamente em decorrência da atuação da água.

Dentre os pontos analisados, o ponto 8 foi um dos que representou maior distinção em comparação aos demais. Essa si- tuação pode ser atribuída ao maior percentual de areia nesse ponto, em comparação aos demais; logo, nesse ponto o solo retém menos água.

\subsection{Determinação das Curvas de Retenção e Condutividade do Solo pelo método Beerkan}

A fim de determinar os parâmetros de forma, assume-se a similaridade com a forma da curva de distribuição do tamanho das partículas $F(D)$. Na Figura 5 é possível observar a curva de distribuição do tamanho das partículas $F(D)$ a partir da média dos 9 pontos onde foram realizados a granulometria do solo em estudo.

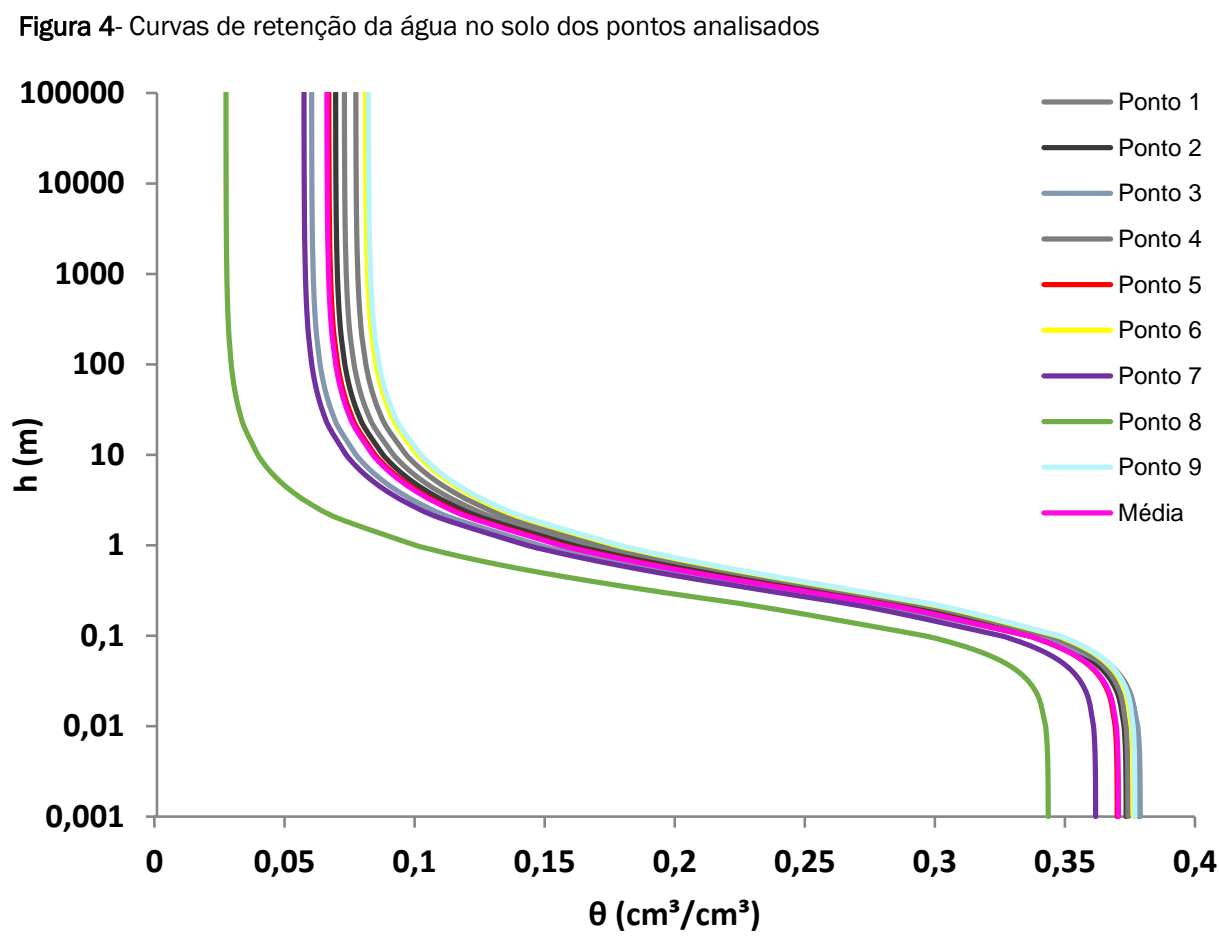

Fonte: Autores

Figura 5- Curva de distribuição do tamanho das partículas $F(D)$.

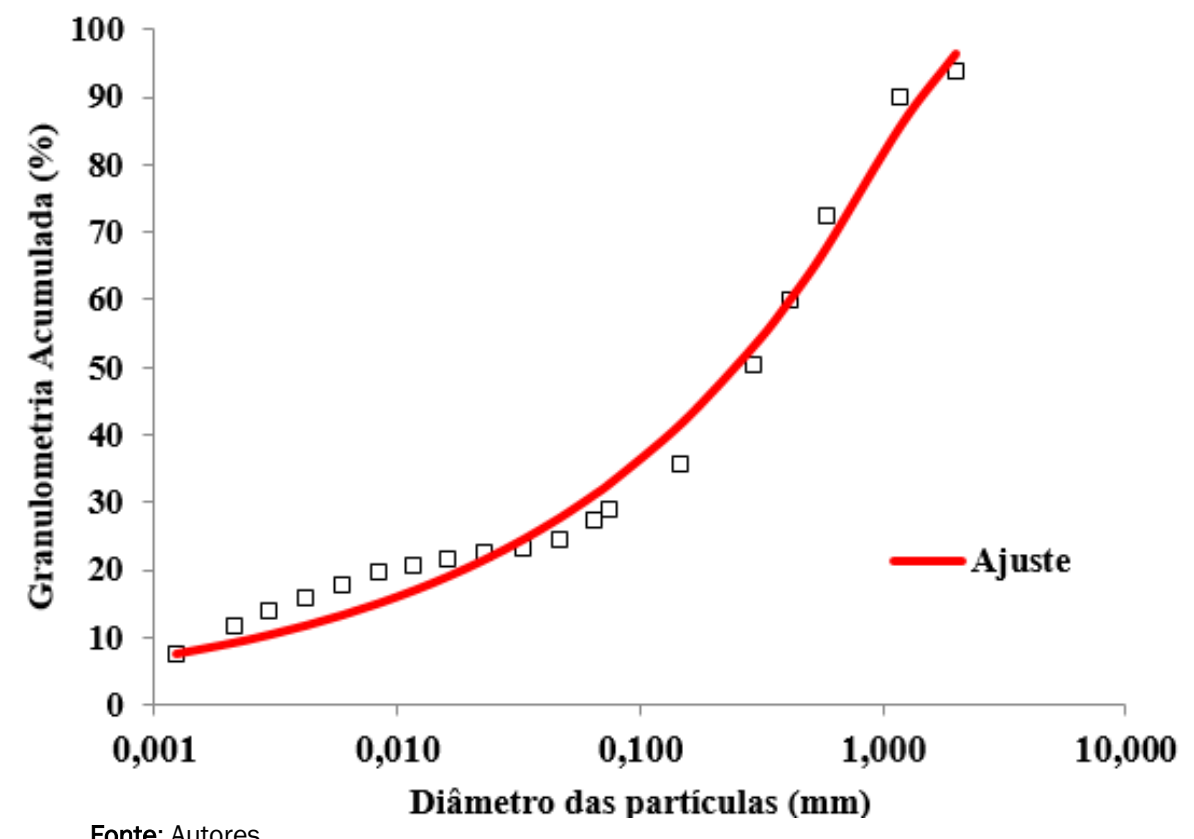


É possível observar na Figura 5, bom ajuste da curva F(D) com a granulometria obtida, com erro de $7,99 \%$, obtido pela raiz quadrada da divisão da soma dos erros entre os valores estimados e medidos.

Com os dados obtidos pelos ensaios de infiltração, foram ge- radas curvas de infiltração acumulada em função do tempo em segundos para tempo curto e tempo longo, para os três pontos analisados. Nas Figura 6 observa-se o ajuste da curva de infiltração para o tempo curto e longo para os pontos 1, 2 e 3, respectivamente.

Figura 6- Curva de infiltração ajustada para o tempo curto e longo nos Pontos 1,2 e 3
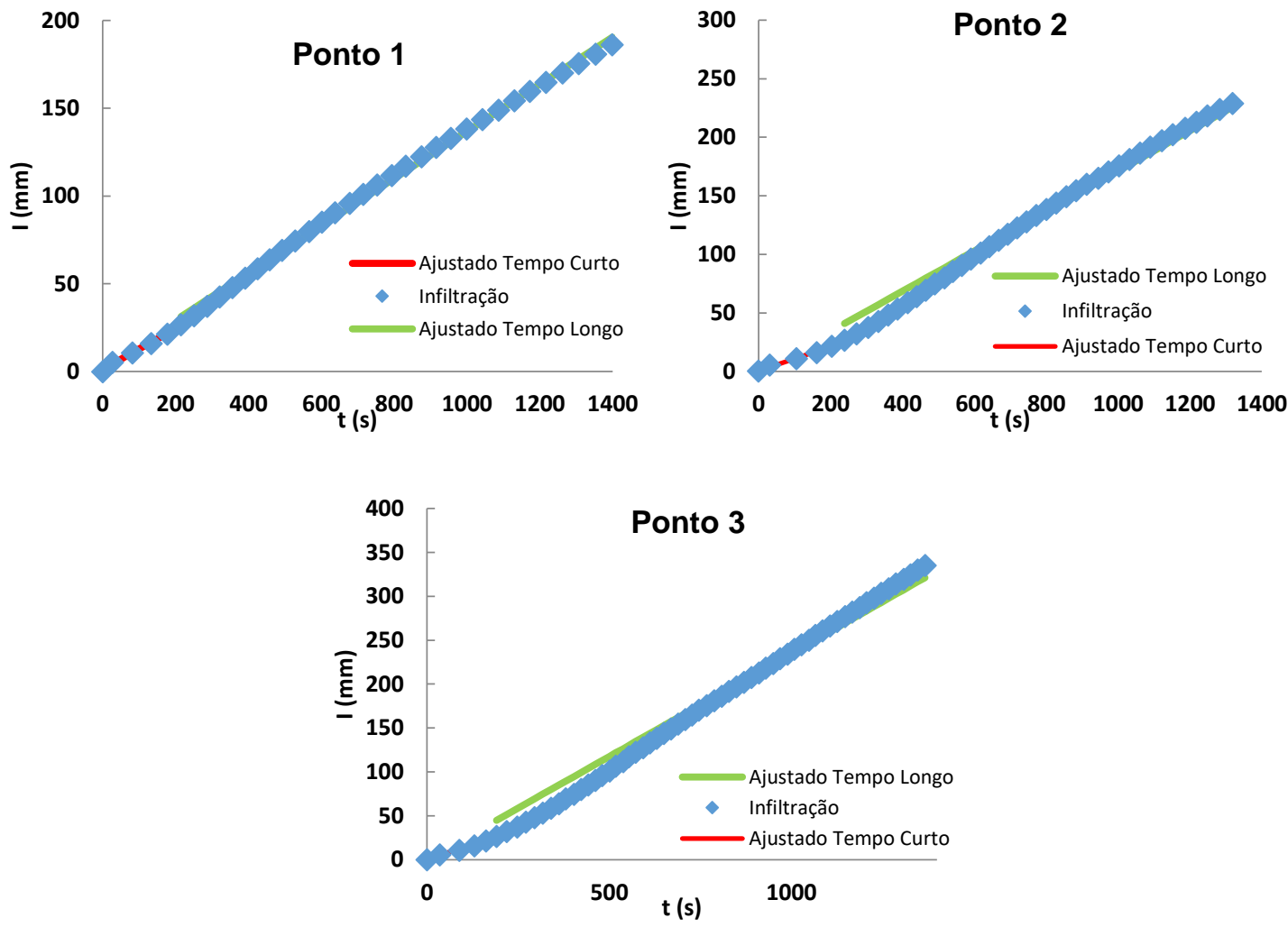

Fonte: Autores

As curvas de tempo curto e tempo longo são estimadas a partir dos dados de infiltração. Conforme estabelecido por Lassabatere et al. (2006), para o tempo curto, fluxo transitório, fo considerado os dados de infiltração até o $5^{\circ}$ ponto, etapa que é obtida a sorvidade. Para o tempo longo, fluxo estacionário, foi considerado do $6^{\circ}$ ponto de infiltração até o tgrav (tempo gravimétrico), etapa que é obtida a condutividade hidráulica saturada, devido a infiltração entrar em estado constante.

É possível observar na Figura 6 a diferença entre os ajustes das curvas para tempo curto e longo em relação a infiltração dos pontos analisados. Os erros para o fluxo transitório dos pontos 1, 2 e 3, respectivamente, foram: 4,07\%, 6,88\% e $8,52 \%$. Os erros para o fluxo estacionário dos pontos 1,2 e 3 , respectivamente, foram: $1,91 \%, 5,07 \%$ e $5,99 \%$. Verifica-se que os ajustes das curvas para o ponto 1 foram melhores em relação aos pontos 2 e 3, sendo justificado pelo menor erro calculado. 0 fluxo transitório está associado a obtenção da sorvidade (S) e o fluxo estacionário está relacionado à obtenção da condutividade hidráulica saturada $\left(\mathrm{K}_{\mathrm{s}}\right)$. Logo, o solver que obteve os valores de $\mathrm{S}$ e $\mathrm{K}_{\mathrm{s}}$ gerou erros menores para o fluxo estacionário, obtido pela raiz quadrada da divisão da soma dos erros entre os valores estimados e medidos.

Em cada um dos três pontos foram realizadas coletas de amostras para a determinação das propriedades físico-hídricas do solo. Essas propriedades foram a umidade inicial $\left(\theta_{0}\right)$, a umidade saturada $\left(\theta_{s}\right)$, a densidade do solo $\left(\rho_{s}\right)$ e a porosidade $(\sigma)$. A Tabela 2 apresenta as propriedades obtidas através da análise dos três pontos em estudo.

Tabela 2 - Parâmetros Físico-Hídricos dos Pontos analisados

\begin{tabular}{ccccc}
\hline Ponto & $\boldsymbol{\rho}_{\boldsymbol{s}}\left(\mathrm{g} / \mathrm{cm}^{\mathbf{3}}\right)$ & $\boldsymbol{\theta}_{\mathbf{0}}\left(\mathrm{cm}^{3} / \mathrm{cm}^{3}\right)$ & $\boldsymbol{\theta}_{\boldsymbol{s}}\left(\mathrm{cm}^{3} / \mathrm{cm}^{3}\right)$ & $\boldsymbol{\sigma}(\%)$ \\
\hline $\mathbf{1}$ & 1,72 & 0,08 & 0,34 & 35 \\
$\mathbf{3}$ & 1,60 & 0,07 & 0,36 & 39 \\
Média & 1,59 & 0,07 & 0,38 & 39 \\
\hline
\end{tabular}

Fonte: Autores

A densidade das partículas $\left(\rho_{p}\right)$ determinada foi de 2,62 $\mathrm{g} / \mathrm{cm}^{3}$ para os três pontos. Segundo Reichardt e Timm (2004) a densidade das partículas depende da constituição do solo e, esta varia relativamente pouco de solo para solo. A densidade das partículas oscila em torno de $2,650 \mathrm{~g} / \mathrm{cm}^{3}$, sendo próximo do valor obtido no estudo. 
Segundo Beutler et al. (2001), a densidade do solo apresenta implicações diretas sobre a porosidade e infiltração de água no solo. Reichardt e Timm (2004) comentam que as densidades dos solos arenosos oscilam entre 1,4 e $1,8 \mathrm{~g} / \mathrm{cm}^{3}$. De acordo com o Hybras (2018), para os solos franco arenosos, o valor médio da densidade do solo é $1,51 \mathrm{~g} / \mathrm{cm}^{3}$. Sendo assim, os resultados obtidos da densidade do solo nesse estudo estão coerentes.

Os valores de $\theta_{\mathrm{s}}$ obtidos nesse trabalho estão próximos aos observados por Souza et al. (2008), que foi de $0,37 \mathrm{~cm}^{3} / \mathrm{cm}^{3}$ para um solo franco arenoso. Bem como, estão em concordância com os resultados apresentados por Souza et al. (2016) para solos com predominância arenosa, $0,37 \mathrm{~cm}^{3} / \mathrm{cm}^{3}$.

De acordo com Reichardt e Timm (2004), a porosidade é afe- tada pelo nível de compactação, ou seja, quanto maior a densidade, menor a porosidade. Analisando os dados para os três pontos na Tabela 2, o ponto 1 obteve a maior densidade do solo, consequentemente a menor porosidade, confirmando o que foi mencionado na literatura.

Os parâmetros de forma da curva de retenção e condutividade hidráulica, para os pontos analisados, são apresentados na Tabela 3, juntamente com os da literatura. Os parâmetros de forma são obtidos em função da textura do solo, ou seja, da granulometria. Os pontos 1, 2 e 3 obtiveram os mesmos valores de parâmetros de forma pois esses pontos estão no centro dos demais pontos onde foram realizadas as coletas das amostras para a realização da granulometria, sendo utilizada a média da granulometria dos 9 pontos para os cálculos.

Tabela 3- Parâmetros de forma da literatura

\begin{tabular}{cccccc}
\hline Referências & Classe Textural & $\mathbf{n}$ & $\mathbf{m}$ & $\mathbf{n}$ & $\mathbf{c}_{\mathrm{p}}$ \\
\hline Autor (2020) & Franco Arenosa & 2,181 & 0,083 & 13,559 & 2,460 \\
Souza et al. (2008) & Franco Arenosa & 2,130 & 0,060 & 18,160 & 2,520 \\
Santos et al. (2012) & Franco Arenosa & 2,420 & 0,174 & 7,757 & 2,110 \\
Santos (2017) & Areia Franca & 2,370 & 0,160 & 8,390 & 2,040 \\
França Neto (2018) & Franco Arenosa & 2,249 & 0,111 & 11,108 & - \\
Sousa (2019) & Franco Arenosa & 2,292 & 0,127 & 9,925 & 2,153 \\
\hline
\end{tabular}

Fonte: Autores

É possivel observar que para os parâmetros n e m, os valores máximos foram do estudo de Santos et al. (2012) e os valores mínimos foram do estudo de Souza et al. (2008), logo, os valores calculados na pesquisa apresentam concordância satisfatória entre os obtidos na literatura contidos na Tabela 3. Para o parâmetro $\eta$, o valor máximo foi do estudo de Souza et al. (2008) e o menor foi do estudo de Santos et al. (2012), sendo assim, o valor calculado na pesquisa mostra concordância satisfatória entre os obtidos em estudos contidos na Tabela 3. É possível observar para o parâmetro n que há uma relativa diferença entre os valores da literatura contidos na Ta- bela 3 , e isso pode ser atribuído às características diferentes dos locais onde foram realizados os ensaios esses estudos. Para o parâmetro $\mathrm{c}_{\mathrm{p}}$, o valor máximo foi do estudo de Souza et al. (2008), e o valor mínimo do estudo de Santos (2017), logo, o valor calculado na pesquisa apresenta concordância satisfatória entre os obtidos na literatura contidos na Tabela 3.

Os parâmetros de normalização, $\mathrm{S}$ (Sorvidade), $\mathrm{K}_{\mathrm{s}}$ (Condutividade Hidráulica Saturada) e $h_{\mathrm{g}}$ (Potencial de Entrada de Ar), referente aos três pontos analisados, são apresentados na Tabela 4 .

Tabela 4- Parâmetros de normalização dos pontos analisados

\begin{tabular}{cccc}
\hline Ponto & $\mathbf{S}\left(\mathrm{mm} / \mathbf{s}^{0,5}\right)$ & $\mathrm{K}_{\mathbf{s}}(\mathrm{mm} / \mathrm{s})$ & $\mathrm{h}_{\mathbf{g}}(\mathrm{mm})$ \\
\hline 1 & 0,317 & 0,118 & $-0,775$ \\
2 & 0,092 & 0,171 & $-0,056$ \\
3 & 0,095 & 0,235 & $-0,042$ \\
Média & 0,168 & 0,175 & $-0,291$ \\
Mediana & 0,095 & 0,171 & $-0,056$ \\
\hline
\end{tabular}

\section{Fonte: Autores}

É possivel observar na Tabela 4 que os parâmetros de normalização do ponto 1 estão distantes, principalmente para $S$ e $h_{g}$. Isso pode ser atribuído ao ensaio de infiltração, ao qual nos momentos iniciais a infiltração no ponto 1 foi mais rápida em comparação aos pontos 2 e 3, e por isso a sorvidade nesse ponto é maior, pois a sorvidade é a capacidade do solo absorver a água nos primeiros instantes da infiltração. 0 parâmetro $h_{\mathrm{g}}$ depende da sorvidade, e por isso está diretamente ligada a ela, sendo assim, o valor foi distante para o ponto $1 \mathrm{em}$ comparação aos demais. Além disso, é possível associar que para o parâmetro $\mathrm{K}_{\mathrm{s}}$, o ponto 3 teve o valor maior, em relação aos pontos 1 e 2. Isso pode ser atribuído ao fator que o ponto 3 é mais próximo do ponto 8 , determinado na granulometria, ao qual nesse local o percentual de areia pode ser maior. Por isso, a condutividade hidráulica saturada pode ter sido maior, pois em locais mais arenosos o $\mathrm{K}_{\mathrm{s}}$ é maior, conduzindo mais rápido a água no solo.

O valor da mediana foi escolhido para comparar com os valores da literatura, mostrados na Tabela 4. Nesse caso, a mediana é mais adequada, pois não é afetada por pontos atípicos, como por exemplo os valores do ponto 1.

Na Tabela 5, são apresentados os valores dos parâmetros de normalização (S, $\mathrm{K}_{\mathrm{s}}$ e $\mathrm{h}_{\mathrm{g}}$ ) obtidos nos estudos de Souza et al. (2008), Santos et al. (2012), Santos (2017), França Neto (2018) e Sousa (2019), comparando-os com os obtidos na pesquisa. 
Tabela 5- Parâmetros de normalização da literatura

\begin{tabular}{ccccc}
\hline Referências & Classe Textural & $\mathbf{S ~ ( m m / \mathbf { s } ^ { 0 , 5 } )}$ & $\mathbf{K}_{\mathbf{s}}(\mathrm{mm} / \mathbf{s})$ & $\mathbf{h}_{\mathbf{g}}(\mathbf{m m})$ \\
\hline Autor (2020) & Franco Arenosa & 0,095 & 0,171 & $-0,056$ \\
Souza et al. (2008) & Franco Arenosa & 0,490 & 0,020 & $-14,500$ \\
Santos et al.(2012) & Franco Arenosa & 1,569 & 0,224 & $-31,650$ \\
Santos (2017) & Areia Franca & 1,620 & 0,060 & $-53,560$ \\
França Neto (2018) & Franco Arenosa & 0,253 & 0,021 & $-9,255$ \\
Sousa (2019) & Franco Arenosa & 0,391 & 0,019 & $-0,462$ \\
\hline
\end{tabular}

Fonte: Autores

É possível observar que para o parâmetro S, o valor máximo foi do estudo de Santos (2017) e o valor mínimo foi do estudo de França Neto (2019), logo, o valor calculado na pesquisa apresenta uma significativa variabilidade em comparação com os obtidos na literatura contidos na Tabela 5. Para o parâmetro $\mathrm{K}_{\mathrm{s}}$, o valor máximo foi do estudo de Santos et al. (2012) e o valor mínimo foi do estudo de Sousa (2019), sendo assim, o valor obtido na pesquisa mostra concordância satisfatória entre os obtidos na literatura contidos na Tabela 5. Em relação ao parâmetro $h_{g}$, o valor máximo foi do estudo de Sousa (2019) e o valor mínimo foi do estudo de Santos (2017), logo, o valor obtido na pesquisa mostra uma significativa variabilidade em comparação com os demais, sendo apenas um pouco mais próximo do estudo de Sousa (2019).

Além disso, as divergências apresentadas nos resultados do estudo analisado em relação à literatura podem ser decorrentes do fato que os estudos de Souza et al. (2008), Santos et al. (2012), Santos (2017) e França Neto (2018) foram realizados em solos não urbanizados. 0 estudo de Sousa (2019) foi realizado em solo urbanizado, condições relativamente semeIhantes ao do estudo em análise, tendo valores mais próximos para o parâmetro $h_{g}$, visto que os demais estudos tiveram valores muito distantes.

Segundo Wouters et al. (2015), em situações de chuva, para solos urbanos quando a água cai, ela é perdida para o escoamento, devido à presença de superfícies impermeáveis, ao passo que para o solo natural a água é melhor retida devido a vegetação. Além disso, a evaporação em solos urbanizados é muito menor que no solo natural. Ou seja, esses são fatores que indicam que os parâmetros hidrodinâmicos do solo podem sofrer interferência se o estudo for realizado em solos urbanos ou naturais. Logo, é necessário criar estratégias para reduzir os impactos que os solos urbanizados causam, como por exemplo, implementar uma infraestrutura urbana mais verde, como a construção de praças para a sociedade.

Com a determinação dos parâmetros de forma e dos parâmetros de normalização, foram geradas curvas de condutividade $K(\theta)$ e de retenção de água no solo $\theta(h)$ para os três pontos analisados. As curvas de retenção de água no solo $\theta(\mathrm{h})$ para os três pontos analisados são apresentadas na Figura 7.

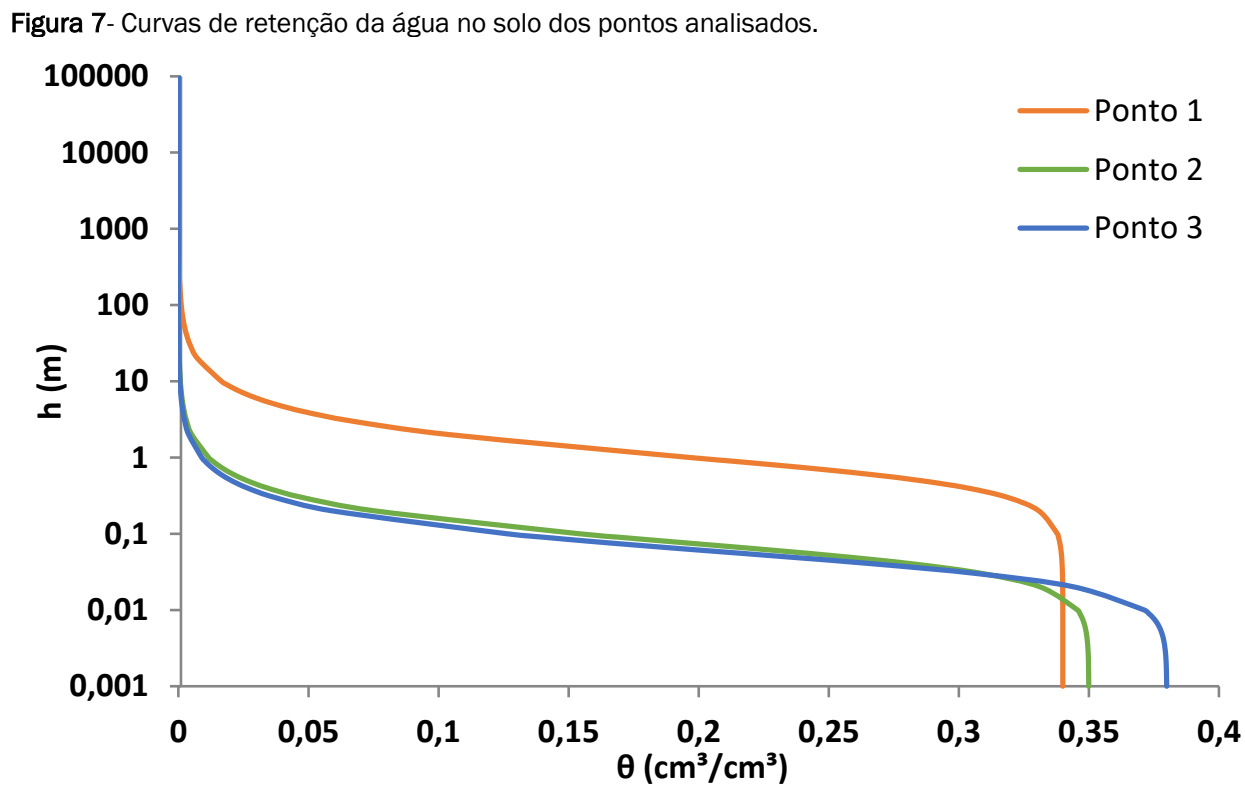

Fonte: Autores

É possível observar na Figura 7 que as curvas têm comportamento relativamente semelhante para os pontos 2 e 3 entre a distribuição das curvas. Observa-se uma pequena variação no teor de umidade saturada e na altura da curva devido a diferença do potencial de entrada de ar $\left(\mathrm{h}_{\mathrm{g}}\right)$ entre os pontos analisados. $\mathrm{O} \mathrm{h}_{\mathrm{g}}$ do ponto 1 foi o maior em módulo dentre os três pontos, por isso teve uma diferença maior, enquanto o $\mathrm{h}_{\mathrm{g}}$ dos pontos 2 e 3 tiveram resultados próximos, sendo semelhantes na inclinação. Como foi explicado anteriormente, o parâmetro $\mathrm{h}_{\mathrm{g}}$ depende da sorvidade, e o ponto 1 foi o que teve maior distinção em comparação com os pontos 2 e 3, por isso, essa diferença maior do ponto 1 na curva de retenção. 
As curvas de condutividade hidráulica do solo $K(\theta)$ para os três pontos analisados são apresentadas na Figura 8.

Figura 8- Curvas de condutividade hidráulica do solo dos pontos analisados.

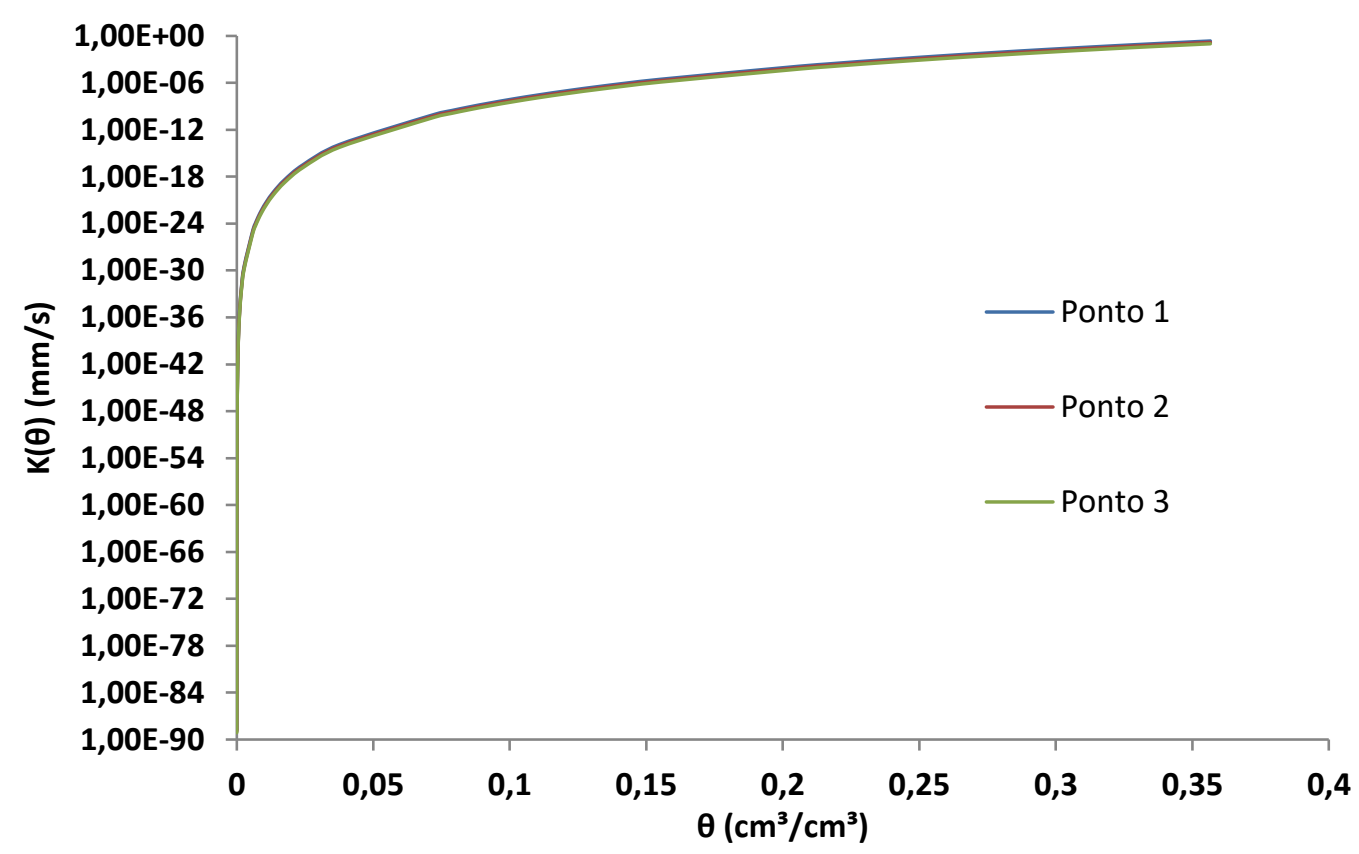

Fonte: Autores

Em relação as curvas de condutividade hidráulica do solo, apresentadas na Figura 8, percebe-se que os três pontos apresentaram comportamento semelhante, devido à semelhança entre as classes texturais dos solos nos pontos analisados.

\section{CONSIDERAÇÕES FINAIS}

De acordo com os ensaios granulométricos, o perfil do solo dos pontos amostrais do Instituto Federal de Pernambuco, Campus Recife, foi classificado como franco arenoso, através dos percentuais de argila, silte e areia. A fim de estimar as propriedades hidrodinâmicas do solo desse local, foram utilizados os métodos da Função de Pedotransferência e Beerkan.

As propriedades hidrodinâmicas do solo, obtidos pelo método da Função de Pedotransferência, foram determinadas a partir dos teores de argila, silte e areia, obtendo-se as curvas de retenção do solo e de condutividade hidráulica do solo, apresentando comportamento semelhante em todos os pontos analisados. Além disso, os parâmetros apresentados estão coerentes com os observados na literatura.

Para o método Beerkan foram determinados os parâmetros de forma ( $m, n, \eta$ e $c_{p}$ ) e os parâmetros de normalização (S, $\mathrm{K}_{\mathrm{s}}$, e $\mathrm{h}_{\mathrm{g}}$ ) da curva de retenção e da curva de condutividade hidráulica do solo estudado, apresentando valores aceitáveis comparados aos da literatura. Além disso, as curvas de infiltração ajustadas, as curvas de retenção e condutividade hidráulica nos pontos analisados tiveram comportamentos semelhantes entre elas.

\section{REFERÊNCIAS}

ANGELOTTI NETTO, A.; FERNANDES, E. J. Condutividade hidráulica de um Latossolo Vermelho em pousio e cultivo intensivo. Pesquisa Agropecuária Brasileira, Brasília, v. 40, p. 797-802, 2005. https://doi.org/10.1590/S0100-204X2005000800010
ASSOCIACAO BRASILEIRA DE NORMAS TECNICAS. NBR 6457: Amostras de solo - Preparação para ensaios de compactação e ensaios de caracterização. Rio de Janeiro, 2016a.

ASSOCIACAO BRASILEIRA DE NORMAS TECNICAS. NBR 7181: Solo - Análise granulométrica. Rio de Janeiro, 2016b.

BARROS, A. H. C.; LIER, Q. DE J. V.; MAIA, A. DE H. N.; SCARPARE, F. V.; Pedotransfer Functions to Estimate Water Rentention Parameters of Soils in Northeastern Brazil. Revista Brasileira de Ciências do Solo, Viçosa, v. 37, n. 2, p379-391, Mar./Apr. 2013. https://doi.org/10.1590/S0100-06832013000200009

BURDINE, N. T. Relative permeability calculations from pore-size distribuition data. Petroleum Transactions, AIME, Dallas, v.198, p.71-77, 1953.

BRUNING, J.; ROBAINA, A. D.; PEITER, M. X.; BOSCAINI, R.; CONCEIÇÃO, C. G.; GOLLO, E. A. Estimativa da curva de retenção de água no solo por função de pedotransferência. Revista de Ciências Agronômicas. Ilha Solteira, SP. v.28, n.1, p.97-110, 2019. ISSN: 24468355.

https://doi.org/10.32929/2446-8355.2019v28n1p97-110

BRAUD, I.; DE CONDAPPA, D.; SORIA, J. M.; HAVERKAMP, R.; ANGULO- JARAMILLO, R.; GALLE, S.; VAUCLIN, M.: Use of scaled forms of the infiltration equation for the estimation of unsaturated soil hydraulic properties (the Beerkan method), Eur. J. Soil Sci., 56, 361-374, 2005.

https://doi.org/10.1111/i.1365-2389.2004.00660.x

BROOKS, R. H.; COREY, A. T. Hydraulic properties of porous media. Hydrology Paper, Colorado State Univ. Fort Collins, v.3, 1964.

CAVALCANTI, G. I. F. N. Efeito da Estrutura nas Propriedades Hidráulicas de Solos do Estado de Pernambuco. Dissertação (Mestrado em Engenharia Civil) - Universidade Federal de Pernambuco, Conselho Nacional de Desenvolvimento Científico e Tecnológico, 2012.

COSTA, L. V. B. ; SOARES, W. A . Desenvolvimento de uma função hidropedológica para umidade volumétrica saturada de solos brasileiros. Holos Environment (Online), v. 19, p. 191, 2019. https://doi.org/10.14295/holos.v19i2.12304 
EMPRESA BRASILEIRA DE PESQUISA AGROPECUÁRIA. EMBRAPA - Manual de Métodos de Análise do Solo. Centro Nacional de Pesquisa de Solos. Rio de Janeiro,1997. ISBN 85-85864-03-6.

FRANÇA NETO, J. M. Variabilidade espacial das propriedades hidrodinâmicas de um solo com vegetação de caatinga. 2018. 85 f. Dissertação (Mestrado) - Universidade Federal de Pernambuco, Programa de Pós-graduação em Engenharia Civil e Ambiental. Caruaru, 2018.

FREDLUND, D.G.; XING, A. Equations for the soil-water characteristic curve. Can Geotech. J., 31, 521-532, 1994. https://doi.org/10.1139/t94-061

FUNASA. Manual de saneamento: orientação técnicas. 3. Ed. Ver Brasília: Fundação Nacional de Saúde, 2006.

GARDNER, W.R. Some steady-state solutions of the unsaturated moisture flow equation with application to evaporation from a water table. Soil Science, [S.I.], v.85, p.228-232, 1958. https://doi.org/10.1097/00010694-195804000-00006

GRIGOLON, G. B. Curva de retenção de água no solo determinada a partir de um número mínimo de pares de umidade e tensão na câmara de Richards. 2013. 83 f. Dissertação (Mestrado) - Escola Superior de Agricultura “Luiz de Queiroz", Piracicaba, 2013.

HAVERKAMP, R.; ROSS, P. J.; SMETTEM, K. R. J.; PARLANGE, J. Y. Three dimensional analysis of infiltration from the disc infiltrometer. Physically based infiltration equation. Water Resources Research, v.30, p.2931-2935, 1994.

https://doi.org/10.1029/94WR01788

HAVERKAMP, R.; BOURAOUI, F.; ANGULO-JARAMILLO, R.; ZAMMIT, C.; DELLEUR, J.W. Soil properties and moisture movement in the unsaturated zone. In: DEULLEUR, J.W. The Handbook of ground water engineering, p. 6.1 - 6.59, 1998.

HOLANDA, M. A. C. R. ; SOARES, W. A . Analysis of the effect of impermeability of urban soils on the infiltration of rainwater in the city of Recife, PE. Revista Ambiente e Agua, v. 14, p. 1, 2019. https://doi.org/10.4136/ambi-agua.2386

JARVIS, N.J.; ZAVATTARO, L.; RAJKAI, K.; REYNOLDS, W.D.; OLSEN, P. A.; MCGECHAN, M.; MECKE, M.; MOHANTY, B.; LEEDS-HARRISON, P.B.; JACQUES, D. Indirect estimation of near-saturated hy draulic conductivity from readily available soil information. Ge oderma, 108, p. 1-17, 2002. https://doi.org/10.1016/S0016$\underline{\text { 7061(01)00154-9 }}$

LASSABATÈRE, L.; ANGULO-JARAMILLO, R.; UGALDE, J. M. S.; CUENCA, R.; BRAUD, I.; HAVERKAMP, R. Beerkan estimation of soi transfer parameters through infiltration experiments - BEST. Soi Science Society of America Journal. Estados Unidos. v. 70, n. 2, p. 521-532, 2006. https://doi.org/10.2136/sssaj2005.0026

MALLANTS, D.; JACQUES, D.; TSENG, P. H.; VAN GENUCHTEN, M.T. FEYEN, J. Comparison of three hydraulic property measurement methods. J. Hydrol. (Amsterdam) 199, p. 295-318, 1997. https://doi.org/10.1016/S0022-1694(96)03331-8
MINASNY, B.; MACBRATNEY, A. B.; BRISTOW, K. L. Comparison of diferente approaches to the development of pedotransfer functions for water-retention curves. Geoderma, v.93, p.225-253, 1999. https://doi.org/10.1016/S0016-7061(99)00061-0

MINASNY, B.; MACBRATNEY, A. B. The efficiency of various approaches to obtain ingestimates of soil hydraulic properties. Geoderma, v.107, p.55 - 70, 2002.

https://doi.org/10.1016/S0016-7061(01)00138-0

MUALEM, Y. A new model for predicting the hydraulic conductivity of unsaturated porous media. Water Resources Research. [S.I.], v. 12, p. 513-522, 1976. https://doi.org/10.1029/WR012i003p00513

RAIMBAULT, G. Diffusivite` et conductivite` hydrauliques de mate' -riaux ou sols non sature' s en eau. Bull. Labo. P. et Ch. 145, p. 125-132, 1986.

REICHARDT, K; TIMM, L C. Solo, planta e atmosfera: conceitos, processos e aplicações. Barueri: Manole, 478 p. 2004.

SANTOS, C. A.G.; SILVA, J. F. C. B. C.; SILVA, R. M. Caracterização hidrodinâmica dos solos da bacia experimental do riacho Guaraíra utilizando o método Beerkan. Revista Brasileira de Recursos Hídricos, v. 17, n. 4 , p. 149-160, 2012

https://doi.org/10.21168/rbrh.v17n4.p149-160

SANTOS, J. Y. G. Avaliação das perdas de água e solo no semiárido paraibano mediante chuva simulada e modelagem hidrossedimentológica. Dissertação (Mestrado). Universidade Federal da Paraíba. Programa de Pós-graduação em Engenharia Civil e Ambiental. Caruaru, 2017.

SOUSA, N. M. Importância das praças na gestão dos recursos hí dricos. Dissertação (Mestrado) - Universidade de Pernambuco, Recife, Pernambuco, 2019.

SOUZA, E. S.; ANTONINO, A. C. D.; ÂNGULO-JARAMILLO, R.; NETTO, A.M. Caracterização hidrodinâmica de solos: Aplicação do método Beerkan. Revista Brasileira de Engenharia Agrícola e Ambiental, Campina Grande, v. 12, p. 128-135, 2008. https://doi.org/10.1590/S1415-43662008000200004

TOMASELLA, J.; HODNETT, M. Pedotransfer functions for tropical soils. Geoderma, v.30, p.415-429, 2004. https://doi.org/10.1016/S0166-2481(04)30021-8

VAN GENUCHTEN, M. Th. A closed-form equation for predicting the hydraulic conductivity of unsaturated soils. Soil Science Society of America Journal, Madison, v.44, p. 892-898, 1980. https://doi.org/10.2136/sssaj1980.03615995004400050002 $\underline{x}$

WAGNER, B.; TARNAWSKI, V.R.; WESSOLEK, G.; PLAGGE, R. Suitability of models for the estimation of soil hydraulic parameters. Geoderma, 86, p. 229-239,1998.

https://doi.org/10.1016/S0016-7061(98)00040-8 